\title{
Identification of cryptic imbalance in phenotypically normal and abnormal translocation carriers
}

\author{
Marzena Gajecka ${ }^{1}$, Caron D Glotzbach ${ }^{1}$, Malgorzata Jarmuz ${ }^{1}$, Blake C Ballif ${ }^{2}$ and \\ Lisa G Shaffer*,1,2
}

${ }^{1}$ Health Research and Education Center, Washington State University Spokane, Spokane, WA, USA; ${ }^{2}$ Signature Genomic Laboratories, LLC, Spokane, WA, USA

Approximately one in 500 individuals carries a reciprocal translocation. Of the 121 monosomy 1 p36 subjects ascertained by our laboratory, three independent cases involved unbalanced translocations of $1 p$ and $9 q$, all of which were designated $t(1 ; 9)(p 36.3 ; q 34)$. These derivative chromosomes were inherited from balanced translocation carrier parents. To understand better the causes and consequences of chromosome breakage and rearrangement in the human genome, we characterized each derivative chromosome at the DNA sequence level and identified the junctions between 1 p36 and 9q34. The breakpoint regions were unique in all individuals. Insertions and duplications were identified in two balanced translocation carrier parents and their unbalanced offspring. Sequence analyses revealed that the translocation breakpoints disrupted genes. This study demonstrates that apparently balanced reciprocal translocations in phenotypically normal carriers may have cryptic imbalance at the breakpoints. Because disrupted genes were identified in the phenotypically normal translocation carriers, caution should be exercised when interpreting data on phenotypically abnormal carriers with apparently balanced rearrangements that disrupt putative candidate genes.

European Journal of Human Genetics (2006) 14, 1255-1262. doi:10.1038/sj.ejhg.5201710; published online 30 August 2006

Keywords: translocation; cryptic imbalance; breakpoint; insertion; duplication

\section{Introduction}

Approximately one in 500 individuals carries a reciprocal translocation. Although balanced translocations are usually associated with normal phenotypes, carriers are at an increased risk of reproductive failure and having offspring with unbalanced rearrangements owing to the abnormal segregation of the rearranged chromosomes. The abnormal phenotype is usually attributed to an imbalance of dosagesensitive genes resulting from the unbalanced karyotype. Although reciprocal translocations are a relatively com-

*Correspondence: Dr LG Shaffer, Health Research and Education Center, Washington State University Spokane, Box 1495, Spokane, WA 992101495, USA. Tel: + 1509368 6710; Fax: + 1509358 7627;

E-mail: Ishaffer@wsu.edu

Received 26 April 2006; revised 20 July 2006; accepted 21 July 2006; published online 30 August 2006 mon class of rearrangements, little is known about the mechanisms that result in translocation formation.

The initial identifications of translocations were made before the use of chromosome banding techniques; these translocations were large and easily distinguished from the normal homolog based on the overall size of the chromosome. With the advent of banding techniques and high-resolution chromosome analysis, more subtle translocations have been identified. The use of molecular cytogenetic technologies such as fluorescence in situ hybridization (FISH) and array-based comparative genomic hybridization (array CGH) have enabled more in-depth characterization of chromosome rearrangements and the identification of cryptic or submicroscopic deletions that are not visible at the current resolution of banded chromosomes. However, few studies have gone beyond 
the level of the light microscope and sequenced the breakpoints of translocations. ${ }^{1-8}$

Our laboratory has been investigating the clinical aspects and the molecular delineation of deletions of $1 \mathrm{p} 36$. Our previous work has shown four classes of rearrangements among subjects with monosomy $1 \mathrm{p} 36$ : terminal deletions, interstitial deletions, unbalanced translocations, and complex rearrangements. Of 121 subject cell lines examined to date, 15 unbalanced translocations have been identified ${ }^{9,10}$ (Shaffer, unpublished data). Of these, four cases involved unbalanced translocations between $1 \mathrm{p} 36$ and $9 \mathrm{q} 34$. Three of these cases are independent and were chosen for the investigation of possible common mechanisms of formation. ${ }^{11}$ During the cloning of the translocation junctions, cryptic imbalance and gene disruptions were identified in the clinically normal, balanced translocation carrier parents.

\section{Subjects and methods Subjects}

We examined 121 subjects with monosomy 1p36 for deletion sizes, parental origin, and classes of rearrangements $^{9,10,12,13}$ (Shaffer, unpublished data). Of these, four subjects, two of which were brother and sister (collectively subject 55), showed unbalanced translocations derived from rearrangements between $1 \mathrm{p} 36$ and $9 \mathrm{q} 34$. These subjects were known to have inherited unbalanced translocations from a balanced translocation carrier parent: subject 55 has a maternally inherited derivative chromosome 1 and subjects 63 and 70 have paternally inherited derivative chromosomes 1 . All three subjects have partial monosomy of $1 \mathrm{p} 36$ and partial trisomy of $9 \mathrm{q} 34$ [der(1)t(1;9)(p36.3;q34.3)].

Subject 55 a is a 3 -year-old Caucasian male born to a 32 year-old G5P2-3, AB2 woman. Several level II ultrasound examinations during the pregnancy showed that he was small for dates. He was born at term by cesarean section secondary to fetal distress. His birth weight was $5 \mathrm{lbs}, 9 \mathrm{oz}$. At 8 weeks of age, he was diagnosed with dilated cardiomyopathy and was placed on digoxin. There was a question of seizures during this time and he was placed on phenobarbital. There is no other history of seizures. He has a history of reflux that could not be controlled by medication, but has had no GI surgeries. At 7 months of age, a brain magnetic resonance imaging (MRI) showed mild to moderate enlargement of the lateral and third ventricles. The white matter myelination was less mature than that expected for a 7 month old, with maturation more typical of a 4- to 5-month-old child. The extra-axial spaces were generous. At age 1 year, he was diagnosed with hypothyroidism. He crawled at 2 years of age, walked at 3 years of age, and has several single words. At 3 years of age, his weight was $14.5 \mathrm{~kg}$ (50th percentile), his height was $93.8 \mathrm{~cm}$ (50th percentile), and his FOC was $47 \mathrm{~cm}(<2$ nd percentile). Facial features included mild brachycephaly, small appearing ears, no dysplasia or asymmetry of the ears, upslanting palpebral fissures, slightly deep-set eyes, flat nose and nasal bridge, and pointed chin. Examination of his extremities revealed short hands and feet, short fifth fingers, and no clinodactyly. He had mild scrotal hypoplasia, with testes high in the scrotum. On neurologic examination at age 3 years, 4 months, he had normal tone. Although an electroencephalogram (EEG) did not show any epileptiform features, superimposed slow activity in the anterior and posterior regions was present. He had a normal swallow function study. His receptive language was equivalent to an 18 month old, and his expressive language was at the 13-month level.

Subject $55 \mathrm{~b}$ is the half-sister of subject $55 \mathrm{a}$. She was born at term. Her birth weight was $6 \mathrm{lbs}, 3 \mathrm{oz}$ (50th percentile). She had tonic-clonic seizures noted at 3 weeks of age. At the time of evaluation at 11 years of age, she was on topamax. On medication, she had about 2-3 seizures per month. She has a history of cardiomyopathy and was treated with digoxin for 2.5 years. At the time of evaluation, she was on synthroid for hypothyroidism. At age 11 years she had generalized hypotonia with intact deep-tendon reflexes, a strong startle reflex, mild scoliosis, and language skills at the 4-month-old level. Her fine motor skills were at the 9-month-old level and gross motor skills at the 10-month-old level. On the Bayley Scale of Infant Development, she was significantly delayed with developmental mental age equivalent of 8 months and 10 months for psychomotor development. The EEG results were abnormal, with poorly defined generalized slow and sharp wave complexes, which may represent an epileptiform pattern. A brain MRI showed mild opening of the sylvian fissures bilaterally and a thickened cortex consistent with polymicrogyria in both cerebral hemispheres in the perisylvian regions. There was absence of the septum pellucidum, dysgenesis of the splenium of the corpus callosum, and dilatation to the atria and occipital horns of the lateral ventricles. This is superimposed on a mild prominence to the third and lateral ventricles. There was undermyelination in the anterior lobes bilaterally and a mild degree of cerebellar atrophy or cerebellar hypoplasia. Auditory brainstem response audiometry showed loss in the $1000-4000 \mathrm{~Hz}$ frequency bilaterally. At 11 years of age, her weight was $31.3 \mathrm{~kg}$ (25th percentile), height $126.5 \mathrm{~cm} \mathrm{(<3rd} \mathrm{percentile),} \mathrm{and} \mathrm{FOC} \mathrm{of} 51.2 \mathrm{~cm}$ (50th percentile). Her facial features included brachycephaly, low hairline, small ears, no apparent hypotelorism, short palpebral fissures, deep-set eyes, prominent nasal bridge, flat nose, and pointed chin. Her extremities showed small hands and feet, short fifth fingers, clinodactyly, and tapered fingers. She was Tanner stage III. She has the same unbalanced translocation derived from her mother's balanced translocation that her half brother (subject 55a) carries. 
Subject 63 is a Caucasian male who had chromosome analysis at 2 weeks of life because of a heart defect (Epstein anomaly), hypotonia, and cryptorchidism (repaired). He rolled over at 18 months, sat independently at 23 months, and crawled at 3 years of age. At 3 years of age, the child did not have seizures, had a normal brain computed tomography (CT) scan, and no apparent hearing loss. His left eye showed astigmatism. At 3 years of age, he was nonverbal but would make recognizable signs when offered food or toys. He did not demonstrate any self-abuse behaviors. At 3 years of age, the subject was $26 \mathrm{lbs}$ (third percentile) and $90 \mathrm{~cm}$ tall (10th percentile). Although the cytogenetic results were apparently normal, he had a subtelomeric FISH analysis at 3 years of age that showed a derivative chromosome 1 [ish $\operatorname{der}(1) \mathrm{t}(1 ; 9)$ (p36.33;q34.3) (1pter-,9qtel+)]. Subsequent cytogenetic and FISH analyses on the parents revealed that the father carries a balanced translocation $\mathrm{t}(1 ; 9)(\mathrm{p} 36.33 ; \mathrm{q} 34.3)$.

Subject 70 is a Caucasian female referred for a history of seizures at age 3.75 months. A two-vessel cord and poor fetal growth were noted during the pregnancy. An amniocentesis was performed and chromosome analysis was reportedly normal. She was born at 40 weeks gestation via cesarean section for transverse breech presentation. Her birth weight was $5 \mathrm{lb}, 5 \mathrm{oz}$ (50th percentile for a 35.5-week gestation). For the first 2 months of life, she had GE reflux and occasional nasal regurgitation. She has a history of seizures, beginning at 3 months of age, and was on phenobarbital at the time of examination. Her brain MRI and CT scan appeared normal. An EEG did not reveal any abnormal activity. She has bilateral exotropia, left ptosis, and her right retina is smaller than her left. She has no known cardiac abnormalities. At 3.75 months of age, her height was $58.5 \mathrm{~cm}$ (25th percentile) and her head circumference was $39.5 \mathrm{~cm}$ (50th percentile). Her anterior fontanel was $3 \mathrm{~cm} \times 3 \mathrm{~cm}$. Her forehead was broad and the right front was protuberant. Her inner canthal distance, outer canthal distance, and interpupillary distance were all within normal range (50th percentile). Her ears were low set, posteriorly rotated, and small (3rd percentile). In addition, she showed clinodactyly and syndactyly of toes 3-5 on the right foot. Although routine chromosome analysis on peripheral blood lymphocytes was reportedly normal, FISH with subtelomere probes identified an unbalanced translocation between chromosomes 1 and 9 [46,XX.ish $\operatorname{der}(1) t(1 ; 9)(p 36.3 ; q 34)]$. Subsequent testing revealed that the father carries a balanced translocation between chromosomes 1 and 9 .

\section{Array CGH analysis}

To determine the sizes of $1 \mathrm{p} 36$ deletions, microarray-based comparative genomic hybridization (array CGH) was performed on subjects 55 and 70 as previously described ${ }^{14}$ using a microarray constructed from 97 clones from a minimal tiling path of the most distal $10.5 \mathrm{Mb}$ of $1 \mathrm{p} 36 .{ }^{10}$
Microarray analysis for subject 63 was performed at Signature Genomic Laboratories, LLC (Spokane, WA, USA) using the SignatureChip ${ }^{\circledR}$ (Figure 1).

\section{FISH analysis}

To confirm the results of the array CGH analysis, FISH was performed with probes corresponding to the breakpoint clones on the 1 p36 contig as previously described. ${ }^{15}$ bacterial artificial chromosome (BAC) probes were selected using the UCSC (http://www.genome.ucsc.edu) and NCBI (http://www.ncbi.nlm.org) genome browsers to identify clones to distal 9q. These clones were used in metaphase FISH to determine the sizes of the $9 \mathrm{q}$ partial trisomies. The breakpoint-containing clones are shown in Table 1.

To confirm the results from array CGH and metaphase FISH, fiber FISH analysis was performed with the breakpoint-containing BACs according to the methods of Rautenstrauss et al. ${ }^{16}$

\section{Generation of somatic cell hybrids}

To identify the 1p36 breakpoints, somatic cell hybrids from each unbalanced subject were generated as previously described. ${ }^{17}$ Somatic cell hybrids containing either the derivative chromosome 1 or the normal chromosome 1 were identified for each subject. Additionally, hybrids were constructed from the transformed lymphoblast cells from the mother of subject 55 . Hybrids containing the derivative chromosome 9 and derivative chromosome 1 were identified after screening with microsatellite markers and FISH as previously described. ${ }^{17}$

\section{STS marker walking}

To identify the 1 p36 breakpoints in each subject, STS markers were designed and polymerase chain reaction (PCR) analyses were performed using hybrid DNA containing the derivative chromosome 1 from each subject. DNA sequence obtained from GenBank (www.ncbi.nlm.nih.gov) corresponding to the genomic region containing the $1 \mathrm{p} 36$ breakpoint of each subject was analyzed. Repetitive elements were masked from the sequence using Repeat Masker (http://www.repeatmasker.org/) and 200-400 bp STS markers were designed from the unmasked unique sequence using Primer 3 (http://frodo.wi.mit.edu/cgi-bin/ primer3/primer3_www.cgi). Breakpoints were narrowed using markers spaced $\sim 20 \mathrm{~kb}$ apart along the length of the chromosome followed by $\sim 5 \mathrm{~kb}$ apart and finally $\sim 1 \mathrm{~kb}$ apart. To prepare for the subsequent TOPO Walker protocol, the breakpoint for each subject was narrowed to $200-300 \mathrm{bp}$. PCR was performed using $50 \mathrm{ng}$ of the subject's hybrid DNA, 10 pmol each primer, $10 \times$ buffer (TaKaRa), $2.5 \mathrm{~mm}$ each dNTP (TaKaRa), and 0.6 U TaKaRa Ex Taq (TaKaRa) in a final volume of $15 \mu$ l. PCR reactions were initially denatured at $94^{\circ} \mathrm{C}$ for $5 \mathrm{~min}$ followed by 35 cycles of denaturation at $94^{\circ} \mathrm{C}$ for $45 \mathrm{~s}$, annealing at 

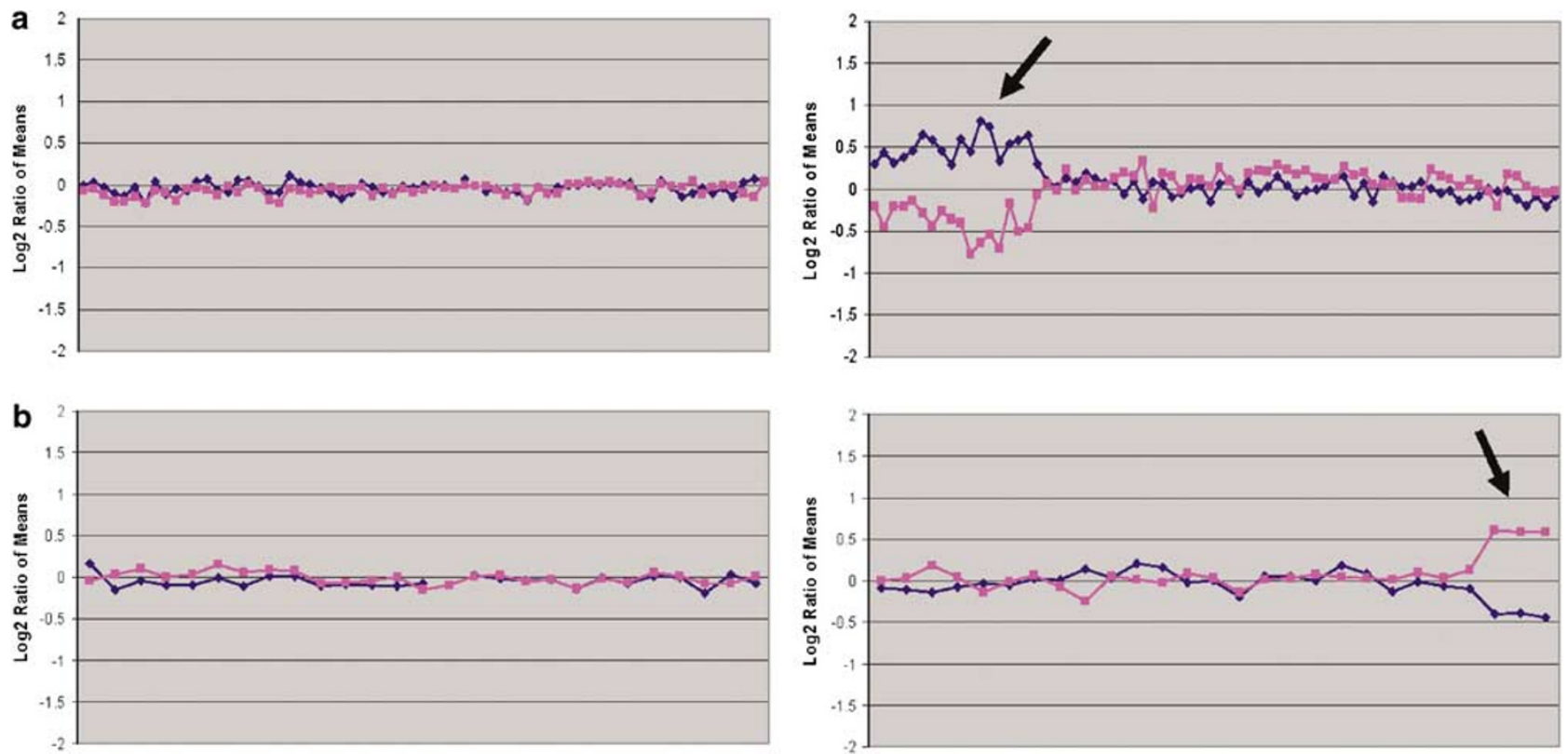

Figure 1 Array $\mathrm{CGH}$ results for subject 63 using the SignatureChip ${ }^{\mathbb{R}}$ targeted array. Each clone on the plot is arranged along the $x$-axis according to its location on the chromosome with the most distal telomeric short-arm clones are on the left and the most distal/telomeric long-arm clones are on the right. The dark blue line represents the control:patient fluorescence intensity ratios for each clone, whereas the pink line represents the fluorescence intensity ratios obtained from a second hybridization in which the dyes have been reversed (patient:control). (a) Detection of a 4.27-Mb deletion on $1 \mathrm{p}$. On the left is a plot for a normal chromosome 1. On the right is a plot for subject 63 showing the deletion. The normal chromosome 1 plot shows a ratio of 0 on a $\log _{2}$ scale for all clones. The plot showing the deletion of $1 p$ shows a significant deviation from 0 for those clones that have a single-copy loss in the individual (arrow). All other clones show a ratio of 0 . (b) Detection of a duplication on chromosome $9 \mathrm{q}$. On the left is a plot for a normal chromosome 9 . On the right is a plot for subject 63 showing the duplication. The plot showing the duplication of $9 q$ shows a significant deviation from 0 for those clones that have a single copy gain in subject 63 .

Table 1 Array CGH and metaphase FISH results

\begin{tabular}{lcccc}
\hline Subject & Deletion size $(\mathrm{Mb})$ & Translocation size $(\mathrm{Mb})$ & 1p36 breakpoint $($ BAC) & 9q34.3 breakpoint $($ BAC) \\
\hline 55 & 6.33 & 3.06 & AL031848 & AL390778 \\
63 & 4.27 & 4.73 & AL591916 & AL590710 \\
70 & 3.46 & 4.58 & AL513320 & AL357934 \\
\hline
\end{tabular}

Deletions sizes estimated in accordance with UCSC Genome Bioinformatics Site (Human March 2006 (hg18) assembly).

$58-59^{\circ} \mathrm{C}$ for $45 \mathrm{~s}$, extension at $72^{\circ} \mathrm{C}$ for $70 \mathrm{~s}$ and a final extension at $72^{\circ} \mathrm{C}$ for $10 \mathrm{~min}$.

\section{TOPO Walker protocol}

The TOPO Walker Kit (Invitrogen, Version E, for isolating and sequencing unknown BAC DNA) was used to amplify and sequence junction fragments in each subject. We used restriction enzymes that are predicted to leave a $3^{\prime}$ overhang based on the sequence near each breakpoint: SacI (subject 55), BglI (subject 63), PstI (subject 70). We followed the standard TOPO Walker protocol with the following modifications. Nested PCR was performed using $0.5 \mu \mathrm{l}$ of 1/1000 diluted initial PCR product, 50 ng LinkAmp Primer 2, 50 pmol GSP3, $10 \times$ buffer (TaKaRa), $2.5 \mathrm{~mm}$ each dNTP (TaKaRa) and $1 \mathrm{U}$ TaKaRa Ex Taq (TaKaRa) in a final volume of $20 \mu \mathrm{l}$. PCR reactions were initially denatured at $94^{\circ} \mathrm{C}$ for
$4 \mathrm{~min}$ followed by 27 cycles of denaturation at $94^{\circ} \mathrm{C}$ for $35 \mathrm{~s}$, annealing at $61^{\circ} \mathrm{C}$ for $35 \mathrm{~s}$, extension at $72^{\circ} \mathrm{C}$ for $1 \mathrm{~min}$, and a final extension at $72^{\circ} \mathrm{C}$ for $4 \mathrm{~min}$.

\section{Junction DNA sequencing}

Gel-purified PCR products from the TOPO Walker protocol were sequenced using an ABI PRISM 3100-Avant genetic analyzer and Big Dye reagents (Applied Biosystems) using previously described methods. ${ }^{18}$ Sequences were localized in the human genome version hg18 using BLAT. ${ }^{19}$ Positions of cytogenetic bands were obtained from the UCSC May 2003 genome annotation database. ${ }^{20}$ RepeatMasker (Smith and Green unpublished; http://www.repeatmasker.org/), Repbase Update libraries, ${ }^{21}$ and TandemRepeatFinder ${ }^{22}$ were used to identify repetitive elements. Sequencher 4.6 (Gene Codes, Ann Arbor, MI, USA) was used to analyze sequence 
$200 \mathrm{bp}$ on either side of the breakpoint junctions to predict intron/exon boundaries and potential fusion transcripts.

\section{Results}

\section{Characterization of rearrangements}

Array CGH was performed to determine the sizes of the 1 p36 deletions. Figure 1 shows an example of array CGH results for the rearrangement in subject 63. A deletion of $4.27 \mathrm{Mb}$ is shown by a loss of DNA as compared to the normal control reference. Likewise, this rearrangement shows a gain of $9 \mathrm{q}$ sequences as compared to the normal control reference. Table 1 presents array CGH and metaphase FISH results confirming the 1p36 deletion sizes and 9q34.3 translocation fragment sizes and the breakpoint-containing BAC for each of the three independent rearrangements. Breakpoint locations are different in all three, with no common $1 p$ deletion or $9 q$ translocation breakpoint.

We performed fiber FISH to confirm our array CGH and metaphase FISH results. Using BAC clones that contained the breakpoints from the 1 p36 and 9q34.3 regions, the junctions were confirmed by visualizing the proximity of the BACs for each rearrangement. Representative fiber FISH results for the junction in the rearrangement found in subjects 55 are shown in Figure 2.

\section{Junction analyses}

STS marker walking analyses using hybrids containing derivative chromosomes 1 allowed us to define the 1 p36 breakpoint in each rearrangement at the sequence level. After narrowing the regions containing the junctions, we used the TOPO Walker protocol to amplify across the junctions. To accomplish this, we used a unique-sequence reverse primer located 200-300 bp from the putative 1 p36 breakpoint and a forward primer LinkAmp Primer 2 (from the TOPO Walker kit). We were able to clone the junction fragments and perform sequence analyses for the junctions between 1p36 and 9q34.3 in each derivative chromosome 1 (Figure 3). Because the balanced translocation carrier parents were available for all three subjects, both the derivative 1 and derivative 9 junctions could be analyzed. On the derivative chromosomes 1 for subjects 55 and 63, 5-bp insertions between the $1 \mathrm{p} 36$ and 9q34.3 fragments were identified. In subjects 55 , the insertion sequence consists of five cytosine bases, whereas in subject 63 , a CACCT sequence was identified at the junction. A simple junction in subject 70 for the derivative 1 was observed with no additional sequence. In each subject, the sequences belonging to either chromosomes 1 or 9 at the cloned and sequenced fragments showed 99-100\% identity to sequences for that respective chromosome using BLAT analysis (http://genome.ucsc.edu/cgi-bin/hgBlat, March 2006 assembly).

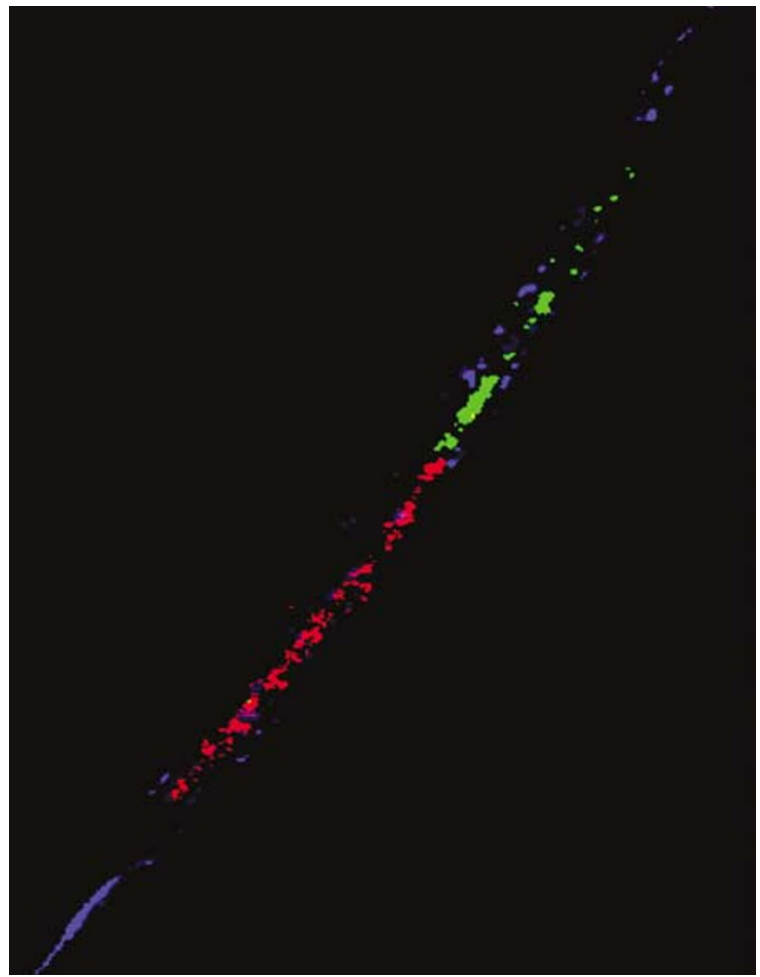

Figure 2 Fiber FISH results for the junction in subject 55 . The probe from 1 p36 breakpoint (AL031848, labeled in green) and the probe from 9q34.3 (AL390778, labeled in red) hybridized at the junction region, confirming the presence of a $1 p 36-9 q 34.3$ BAC junction.

Using cell lines from the carrier mother of subjects 55, the carrier father of subject 63 , and the carrier father of subject 70, the junctions at the derivative chromosomes 1 were confirmed and the junctions at the chromosomes 9 were analyzed. The same CCCCC insertion identified on the derivative chromosome 1 in both subjects 55 was found in the balanced carrier mother (Figure 3a). In addition, a 5-bp (TGGGG) fragment was found at both junctions on the derivative 9 and derivative 1 chromosomes, resulting in a cryptic duplication in the balanced carrier mother. Thus, this phenotypically normal individual has a cryptic imbalance resulting in a 5-bp duplication and 5-bp insertion. Similarly, the junction at the derivative 9 in the father of subject 63 was cloned and sequenced, revealing a similar cryptic imbalance in the phenotypically normal father (Figure $3 \mathrm{~b}$ ). The CACCT insertion found in subject 63 was confirmed in the balanced carrier father, and a 4-bp duplication $(C A A G)$ on both the derivative 1 and derivative 9 was identified. No duplication or insertion of DNA sequence at the junctions in subject 70 and his father were observed (Figure 3c).

To exclude the possibility that the five-nucleotide insertions represent discrepancies in the genome database sequences, the normal chromosomes 1 and 9 were 


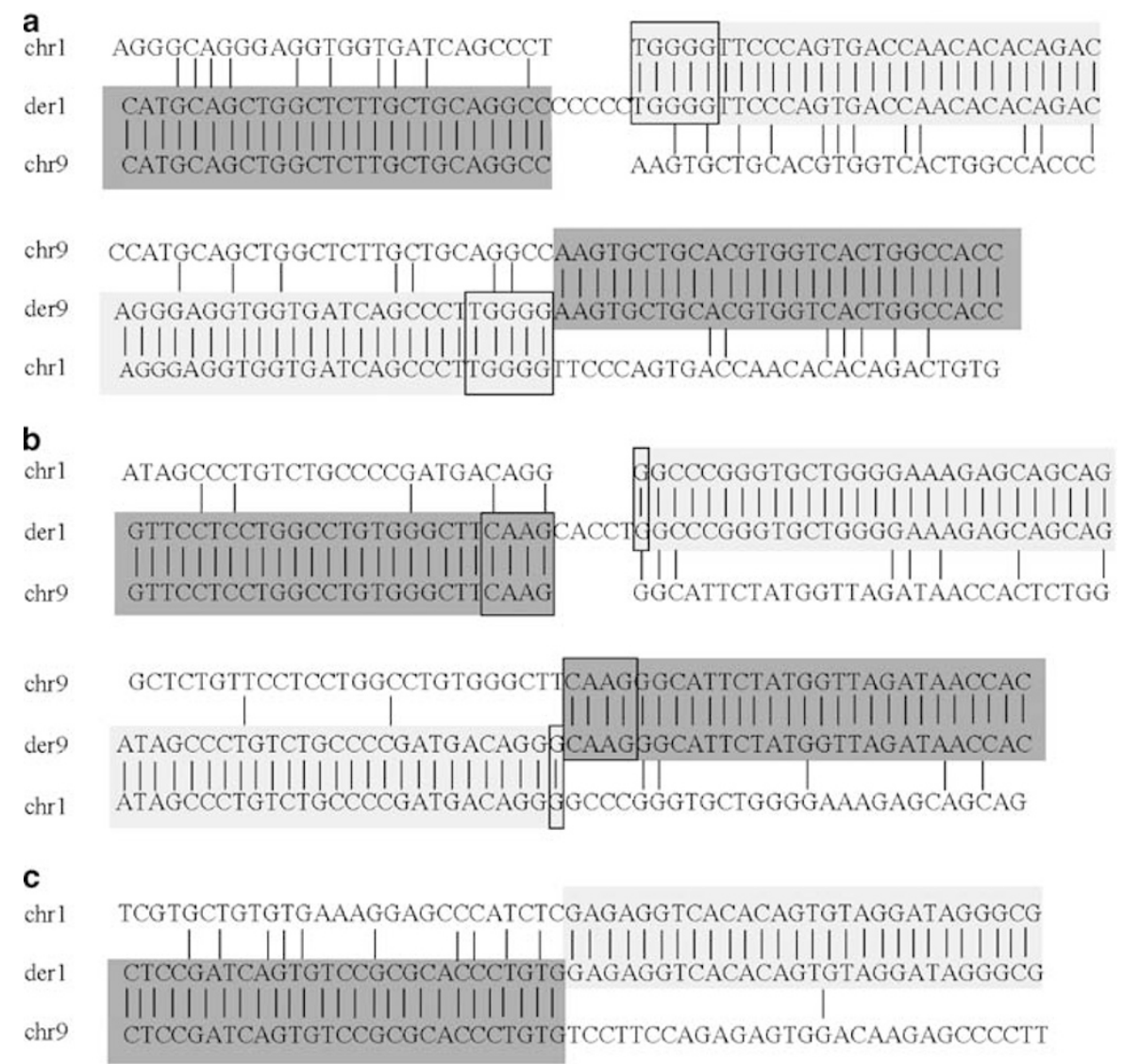

Figure 3 Sequence analysis of the translocation breakpoints. This figure shows the sequence analyses for the junctions between $1 p 36$ and $9 q 34.3$ in each derivative chromosome for the three subjects and their translocation carrier parents. Light gray boxes indicate chromosome 1 sequence. Dark gray boxes indicate chromosome 9 sequence. Black-outlined boxes indicate duplicated sequence. (a) Subjects 55 and carrier mother. (b) Subject 63 and carrier father. (c) Subject 70 and carrier father.

Table 2 Genes interrupted by translocation breakpoints in three $t(1 ; 9)$ subjects

\begin{tabular}{lll}
\hline Subject & $1 p 36$ breakpoint & Gene \\
\hline 55 & acyl-CoA thioesterase 7 (ACOT7) (OMIM \#602587) & $9 q 34.3$ breakpoint \\
63 & Chr1_112.1 (Geneid gene prediction) & AK127261 \\
70 & Epidermal growth factor (EGF)-like-domain multiple 3 (EGFL3) & Sarcosine dehydrogenase (SARDH) \\
& (OMIM \#604266) & Oncogene VAV2 (VAV2) (OMIM \\
& & \#600428) \\
\hline
\end{tabular}

sequenced at these locations in the mother and father of subjects 55 and 63, respectively, and five unrelated control individuals. The normal chromosomes did not show these 5-bp insertions, making it unlikely that they represent normal sequence variations.

The junction fragment analysis revealed that the breakpoints on $1 \mathrm{p}$ and $9 \mathrm{q}$ each interrupt a known or predicted gene in all rearrangements. Four breakpoints were identified in known genes and two breakpoints were identified in predicted genes (Table 2). Thus, because the breakpoints and insertions disrupt known or predicted genes, this provides further support that the observed insertions are unlikely to represent normal genomic variation.

To predict whether the subjects' breakpoint junctions might produce fusion transcripts, we examined the putative products of each subject's breakpoint junction. In cases 63 and 70, the genes are transcribed in the opposite orientations. In these cases, transcription of a fusion gene is probably aborted. In the case of subject 55, both genes are transcribed in the same orientation but in different frames. A potential transcript might be produced, but the frameshift introduces premature termination 
codons less than $200 \mathrm{bp}$ from the breakpoint junctions in both the derivative 1 and derivative 9, and such transcripts would be sensitive to nonsense-mediated RNA decay. Thus, we predict that no stable proteins are likely produced in any of the subjects from the derivative chromosomes.

\section{Discussion}

A direct correlation between genotype and phenotype is typically assumed for unbalanced translocations, the abnormal phenotyped attributed to either (1) an imbalance of dosage-sensitive genes caused by the gain or loss of one or both chromosome segments involved, (2) the disruption of a gene by a translocation breakpoint, or (3) the formation of a novel fusion gene product. Thus, for the $6 \%$ of balanced translocations that correspond with an abnormal phenotype, ${ }^{23}$ it has been inferred that either the balanced translocation is not, in fact, balanced but contains duplications or deletions too small to be observed by cytogenetic means ${ }^{24-26}$ or the translocation is indeed balanced but one or more breakpoints disrupts a gene. ${ }^{25}$ Recently, the breakpoints of several apparently balanced translocations in phenotypically abnormal carriers have been analyzed using molecular cytogenetic techniques, revealing the presence of small cryptic duplications and deletions. Gribble et $a l^{26}$ used array CGH to characterize apparently balanced translocations in individuals with abnormal phenotypes. Because array CGH will not identify balanced rearrangements as abnormal, the group used flow-sorted chromosomes to separate the two derivative chromosomes and performed array CGH on each derivative chromosome. This resulted in the identification of three of 10 cases with complex rearrangements involving deletions, inversions, and insertions at or near the breakpoints. ${ }^{26}$ As a negative confirmation of their hypothesis, the same group ${ }^{27}$ examined 13 phenotypically normal individuals with apparently balanced reciprocal translocations using FISH and array CGH and reported that none had cryptic imbalance at the resolution of BACs, approximately $150 \mathrm{~kb}$. Although they did not discover any insertions or deletions, consistent with their hypothesis, two breakpoints bisected known genes, which were larger than the breakpoint-containing BACs and were necessarily disrupted. ${ }^{27}$

In contrast, we found cryptic imbalances in two carrier parents with apparently balanced translocations and normal phenotypes. This finding supports the suggestion that the abnormal clinical features observed in our unbalanced subjects are the result of partial monosomy of 1p36/partial trisomy of 9q34 rather than disruption of these genes. Further implications of our results are that the finding of imbalances and gene disruptions in apparently balanced translocation carriers with phenotypic abnormalities should be interpreted with caution because we have shown that this can be found in clinically normal individuals.

The present study also illustrates the necessity of examining breakpoints in translocation carriers at the sequence level. Although Batista et $\mathrm{al}^{27}$ identified disruption of genes in two of 13 cell lines, this number may be artificially low because they were able to identify gene breakage only if the genes circumscribed the breakpointcontaining BACs. Because BACs are, on average, 100$150 \mathrm{~kb}$, any smaller disruption would be missed. Indeed, the 5-bp insertions in our study were not detected by FISH or array CGH and only could be detected after cloning the translocation breakpoints.

\section{Acknowledgements}

We thank Aaron Theisen (Washington State University, Spokane, WA) for his critical editing of the manuscript and Adam Pavlicek (Genetic Information Research Institute, Mountain View, CA, USA) for examining the breakpoint junctions of the subjects.

\section{References}

1 Mansouri MR, Carlsson B, Davey E et al: Molecular genetic analysis of a de novo balanced translocation $\mathrm{t}(6 ; 17)(\mathrm{p} 21.31 ; \mathrm{q} 11.2)$ associated with hypospadias and anorectal malformation. Hum Genet 2006; 119: 162-168.

2 Bodrug SE, Holden JJ, Ray PN, Worton RG: Molecular analysis of $\mathrm{X}$-autosome translocations in females with Duchenne muscular dystrophy. EMBO J 1991; 10: 3931-3939.

3 Bodrug SE, Ray PN, Gonzalez IL, Schmickel RD, Sylvester JE, Worton RG: Molecular analysis of a constitutional X-autosome translocation in a female with muscular dystrophy. Science 1987; 237: $1620-1624$.

4 Haider S, Matsumoto R, Kurosawa N, Wakui K, Fukushima Y, Isobe M: Molecular characterization of a novel translocation $\mathrm{t}(5 ; 14)(\mathrm{q} 21 ; \mathrm{q} 32)$ in a patient with congenital abnormalities. J Hum Genet 2006; 51: 335-340.

5 McMullan TW, Crolla JA, Gregory SG et al: A candidate gene for congenital bilateral isolated ptosis identified by molecular analysis of a de novo balanced translocation. Hum Genet 2002; 110: $244-250$.

6 Millar JK, Wilson-Annan JC, Anderson S et al: Disruption of two novel genes by a translocation co-segregating with schizophrenia. Hum Mol Genet 2000; 9: 1415-1423.

7 Schule B, Albalwi M, Northrop E et al: Molecular breakpoint cloning and gene expression studies of a novel translocation $\mathrm{t}(4 ; 15)(\mathrm{q} 27 ; \mathrm{q} 11.2)$ associated with Prader-Willi syndrome. BMC Med Genet 2005; 6: 18.

8 Yoshiura K, Machida J, Daack-Hirsch S et al: Characterization of a novel gene disrupted by a balanced chromosomal translocation $\mathrm{t}(2 ; 19)$ (q11.2; 13.3$)$ in a family with cleft lip and palate. Genomics 1998; 54: 231-240.

9 Ballif BC, Kashork CD, Shaffer LG: FISHing for mechanisms of cytogenetically defined terminal deletions using chromosomespecific subtelomeric probes. Eur J Hum Genet 2000; 8: 764-770.

10 Heilstedt HA, Ballif BC, Howard LA et al: Physical map of 1p36, placement of breakpoints in monosomy 1p36, and clinical characterization of the syndrome. Am J Hum Genet 2003; 72: $1200-1212$.

11 Gajecka M, Pavlicek A, Glotzbach CD et al: Identification of sequence motifs at the breakpoint junctions in three $\mathrm{t}(1 ; 9)(\mathrm{p} 36.3 ; \mathrm{q} 34)$ and delineation of mechanisms involved in generating balanced translocations. Hum Genet 2006, (in press). 
12 Shapira SK, McCaskill C, Northrup H et al: Chromosome 1p36 deletions: the clinical phenotype and molecular characterization of a common newly delineated syndrome. Am J Hum Genet 1997; 61: $642-650$.

$13 \mathrm{Wu}$ YQ, Heilstedt HA, Bedell JA et al: Molecular refinement of the 1p36 deletion syndrome reveals size diversity and a preponderance of maternally derived deletions. Hum Mol Genet 1999; 8: $313-321$

14 Yu W, Ballif BC, Kashork CD et al: Development of a comparative genomic hybridization microarray and demonstration of its utility with 25 well-characterized 1 p36 deletions. Hum Mol Genet 2003; 12: $2145-2152$.

15 Shaffer LG, McCaskill C, Han JY et al: Molecular characterization of de novo secondary trisomy 13. Am J Hum Genet 1994; 55: 968-974.

16 Rautenstrauss B, Fuchs C, Liehr T, Grehl H, Murakami T, Lupski JR: Visualization of the CMT1A duplication and HNPP deletion by FISH on stretched chromosome fibers. J Peripher Nerv Syst 1997; 2: 319-322.

17 Page SL, Shaffer LG: Nonhomologous Robertsonian translocations form predominantly during female meiosis. Nat Genet 1997; 15: $231-232$.

18 Ballif BC, Wakui K, Gajecka M, Shaffer LG: Translocation breakpoint mapping and sequence analysis in three monosomy $1 \mathrm{p} 36$ subjects with $\operatorname{der}(1) \mathrm{t}(1 ; 1)(\mathrm{p} 36 ; \mathrm{q} 44)$ suggest mechanisms for telomere capture in stabilizing de novo terminal rearrangements. Hum Genet 2004; 114: 198-206.

19 Kent WJ: BLAT - the BLAST-like alignment tool. Genome Res 2002; 12: $656-664$.
20 Karolchik D, Baertsch R, Diekhans M et al: The UCSC Genome Browser Database. Nucleic Acids Res 2003; 31: 51-54.

21 Jurka J: Repbase update: a database and an electronic journal of repetitive elements. Trends Genet 2000; 16: 418-420.

22 Benson G: Tandem repeats finder: a program to analyze DNA sequences. Nucleic Acids Res 1999; 27: 573-580.

23 Warburton D: De novo balanced chromosome rearrangements and extra marker chromosomes identified at prenatal diagnosis: clinical significance and distribution of breakpoints. Am J Hum Genet 1991; 49: 995-1013.

24 Wirth J, Nothwang HG, van der Maarel S et al: Systematic characterisation of disease associated balanced chromosome rearrangements by FISH: cytogenetically and genetically anchored YACs identify microdeletions and candidate regions for mental retardation genes. J Med Genet 1999; 36: $271-278$.

25 Astbury C, Christ LA, Aughton DJ et al: Detection of deletions in de novo 'balanced' chromosome rearrangements: further evidence for their role in phenotypic abnormalities. Genet Med 2004; 6 : $81-89$.

26 Gribble SM, Prigmore E, Burford DC et al: The complex nature of constitutional de novo apparently balanced translocations in patients presenting with abnormal phenotypes. J Med Genet 2005; 42: 8-16.

27 Baptista J, Prigmore E, Gribble SM, Jacobs PA, Carter NP, Crolla JA: Molecular cytogenetic analyses of breakpoints in apparently balanced reciprocal translocations carried by phenotypically normal individuals. Eur J Hum Genet 2005; 13: $1205-1212$. 